\title{
Effects of bladder distension on dose distribution of vaginal vault brachytherapy in patients with endometrial cancer
}

\author{
Ozan C. Guler, MD, Cem Onal, MD, Ibrahim Acibuci, MSc \\ Department of Radiation Oncology, Baskent University Faculty of Medicine, Adana, Turkey
}

\begin{abstract}
Purpose: To investigate dosimetric effects of bladder distention on organs at risk (OARs) during treatment of endometrial cancer using 3D image-based planning of postoperative vaginal vault brachytherapy (BRT).

Material and methods: Fifteen patients with early-stage endometrial cancer were studied, each undergoing adjuvant BRT of vaginal vault via $3.5 \mathrm{~cm}$ diameter cylinder. As treatment, $25 \mathrm{~Gy}$ in 5 fractions were delivered to $5 \mathrm{~mm}$ depth of the vaginal mucosa. Dose-volume histograms of OARs were generated individually with bladder empty and with bladder inflated by sterile saline $(180 \mathrm{ml})$, to compare doses received.

Results: Bladder distention appreciably impacted dosimetry of bladder, sigmoid colon, and small bowel, but dosimetry of rectum was unaffected. With bladder inflated, mean cylinder-to-bowel distance increased significantly $(1.69 \mathrm{~cm}$ vs. $1.20 \mathrm{~cm} ; p=0.006)$. Mean minimum dose to most exposed $2 \mathrm{cc}\left(\mathrm{D}_{2 \mathrm{cc}}\right)$ volume also rose significantly at bladder (5.40 Gy vs. 4.55 Gy [18.7\%]; $p<0.001)$, as opposed to near-significant reductions in $\mathrm{D}_{2 \mathrm{cc}}$ at sigmoid colon (15.1\%; $p=0.11)$ and at small bowel $(10.5 \% ; p=0.14)$. A full bladder had no effect on dose to $50 \%$ volume $\left(\mathrm{D}_{50 \%}\right)$ of bladder or rectum, and declines seen in mean $\mathrm{D}_{50 \%}$ values of sigmoid colon $(22.7 \% ; p=0.12)$ and small bowel $(19.0 \% ; p=0.13)$ again fell short of statistical significance.

Conclusions: The combination of a full bladder and an empty rectum may cause significant unwanted increases in BRT dosing of bladder, without significantly impacting sigmoid colon and small bowel exposures. These findings should be validated through further clinical studies.
\end{abstract}

Key words: bladder distention, brachytherapy, dosimetry, endometrial cancer, vaginal vault.

\section{Purpose}

Endometrial cancer is the most common gynecologic malignancy and is largely diagnosed in early stages $[1,2]$. The primary means of treating localized disease is total abdominal hysterectomy and bilateral salpingo-oophorectomy. For all but low-risk tumors, adjuvant radiotherapy (RT) is thought to reduce chances of loco-regional relapse [3], which develops in $10-15 \%$ of patients with early-stage disease (chiefly as vaginal vault recurrence). Adjuvant treatment generally consists of either external beam RT (ERBT) or internal brachytherapy (BRT) [3]. In recent studies, especially in early stage endometrial carcinoma, EBRT has shown no advantage over BRT, and EBRT carries a greater risk of complications [4]. The PORTEC-II (Postoperative Radiation Therapy for Endometrial Carcinoma) study, a randomized trial of adjuvant RT for endometrial cancer, confirmed that vaginal BRT is effective and does not decrease quality of life as much as EBRT, leading to an upsurge in the use of high dose rate (HDR) vaginal vault BRT [3-5]. In the PORTEC-II trial compared with EBRT, reports of toxicity due to vaginal vault BRT are fewer, although currently does indicate a $12.6 \%$ rate of Grade 1 and 2 gastrointestinal side effects with some Grade 3 events $(<1 \%)$ emerging as late complications [5].

Most of the data published lately on vaginal vault BRT stem from $2 \mathrm{D}$ plain film-derived conventional plans. The chief drawback of this approach is that doses to adjacent organs, mainly rectum and bladder, may be inadequately assessed. With the advent of computed tomography (CT)based conformal plans, prescribed doses to target volumes and collateral exposure to bladder and rectum are clearly identified [6,7]. However, the International Commission on Radiation Units and Measurements (ICRU) defines only bladder and rectal reference points in 2D plans, which may not represent actual 3D dose distributions. Even more worrisome is the fact that doses to small bowel are simply ignored in conventional BRT plans, despite the close proximity of vaginal cylinder.
Address for correspondence: Cem Onal, MD, Department of Radiation Oncology, Baskent University Faculty of Medicine, Adana Research and Treatment Centre, 01120 Adana, Turkey, phone: +90-322-3444444/1304, fax: +90-322-3444445, 凶 e-mail: hcemonal@hotmail.com
Received: 23.07.2014

Accepted: 23.11 .2014 Published: 30.12 .2014 
Many studies have evaluated the use of CT-based conformal plans in patients with cervical and endometrial cancers [6-9], although dosimetric parameters of 2D and 3D BRT plans were being compared. The GEC-ESTRO Brachytherapy Working Group has recommended contouring guidelines, concepts, and terms in three-dimensional magnetic resonance image-based treatment planning in cervical cancer BRT, with reports confirming the safety, feasibility, definite advantages, clinical outcome, and late toxicities $[10,11]$. The impact of bladder filling on dose distribution has likewise been amply studied in the context of cervical cancer and endometrium cancer, with conflicting results [9,12-15]. Still, Hung et al. [16] have made a formal claim that bladder distention reduces BRT dose to small bowel in patients with endometrial cancer. Kobzda et al. [15] found that the dose to the empty bladder was lower than when the bladder was full, and the doses to the bowels increased proportionally in the empty state of the bladder comparing to the full organ. Despite the abundance of data on bladder distention during BRT with the uterus intact, BRT delivery via vaginal cylinder is seldom a focus of 3D image-based dosimetric studies.

The purpose of this study was to investigate the dosimetric effects of bladder distention on organs at risk (OARs) during treatment of endometrial cancer (i.e., bladder, rectum, sigmoid colon, and small bowel) using $3 \mathrm{D}$ image-based planning of postoperative HDR vaginal vault BRT.

\section{Material and methods \\ Patients}

Between November 2013 and January 2014, a total of 15 consecutive patients with early-stage endometrial cancer were recruited for study, each of whom submitted to postoperative adjuvant BRT of vaginal vault. All had undergone total abdominal hysterectomy, and none had received EBRT prior to BRT. Approval was obtained from the institutional review board for this outcome analysis.

Before delivery of BRT, a detailed gynecologic examination was performed to evaluate vaginal vault and to determine applicator diameter. A laxative or enema purge of intestinal contents was also required in advance. Intracavitary BRT was achieved by using plastic, CT-compatible applicators to position standard $3.5 \mathrm{~cm}$ diameter cylinders. After insertion of cylinder to the top of the vagina, the applicator was fixed with a universal applicator clamping device (Varian Medical Systems, Inc., Palo Alto, CA, USA), which was underneath the patient. The length of the cylinder protruding outside the vagina was noted to ensure accurate repositioning for subsequent insertions. The same applicators were utilized throughout the course of treatment. Additionally, before each BRT sessions, the applicator was checked with fluoroscopy whether it was at the same position as the first application. A CT scan with $2.5 \mathrm{~mm}$ slice thickness through the pelvis was carried out at the first BRT application in line with departmental policy.

\section{Treatment planning}

Prior to BRT, a Foley catheter was inserted, with $7 \mathrm{ml}$ of contrast material filling the balloon. Two CT scans were then performed following BRT, leaving in place the secured vaginal cylinder applicator. The initial CT scan was done with an empty bladder, thereafter infusing sterile saline $(180 \mathrm{ml})$ via catheter. A metal clamp was placed to prevent voiding, and second CT scan was done with the bladder full. The pelvis was scanned from lumbosacral junction to ischial tuberosity at $2.5 \mathrm{~cm}$ slice thickness. All CT slices were ultimately transferred to the 3D treatment planning system (BrachyVision ${ }^{\mathrm{TM}}$ Eclipse; Varian Medical Systems, Palo Alto, CA, USA).

The dose was prescribed to $5 \mathrm{~mm}$ depth for the cranial 3 to $5 \mathrm{~cm}$ of the vagina, and the dose delivered during the treatment was 25 Gy in 5 fractions. Vaginal length was measured on CT images, and two-thirds of the vaginal cylinder was routinely activated, in accordance with our institutional protocol.

\section{Organs at risk}

Bladder, rectum, sigmoid colon, and small bowel constituted OARs. In each axial CT slice, external pelvic contours of bladder (empty or full), rectum, sigmoid colon, and small bowel were delineated by the treatment planning system, using sagittal and coronal views to supplement. Oral or intravenous contrast was not used during planning CT. Rectum was defined as the colonic segment between rectosigmoid junction and anal verge [17]. The length of bowel extending proximal to rectum and delimited by a transition to vertical orientation was considered sigmoid colon. Small bowel encompassed the remaining individual intestinal loops, up to the level of the inferior sacroiliac joints (excluding rectum and sigmoid colon), as was previously defined [12]. To ensure structural consistency of patient scan sets (with bladder empty and full), contours of rectum, sigmoid colon, and small bowel were examined in corresponding CT slices to verify that bony landmarks and axial sections of organs closely matched.

For assessment of the distance between the cylinder applicator and bowel, the cylinder-to-bowel distance was defined as the shortest distance from the cylinder apex to the contoured sigmoid or small bowel. Distances were measured by use of the sagittal slice through the midplane of the cylinder, by a single investigator to minimize the risk of interobserver variation.

\section{Statistical analysis}

Statistical analysis relied on standard software (SPSS v20.0; SPSS Inc., Chicago, IL, USA). Volumes of all OARs specified were determined, and dose-volume histograms generated in each bladder state were compared. Volume dose values were expressed as minimum doses to most exposed 0.1-, 0.2-, 0.5-, 1.0-, and 2.0-cc volumes $\left(\mathrm{D}_{0.1 \mathrm{cc}}\right.$ to $\left.\mathrm{D}_{2 \mathrm{cc}}\right)$ and dose received by $50 \%$ of OAR volume $\left(\mathrm{D}_{50 \%}\right)$. In addition, OAR dosing minimum $\left(\mathrm{D}_{\min }\right)$, maximum $\left(\mathrm{D}_{\max }\right)$, mean $\left(\mathrm{D}_{\text {mean }}\right)$, and median $\left(\mathrm{D}_{\text {median }}\right)$ values were calculated for both plans. The Wilcoxon matched-pairs test was applied to 
identify statistical differences in volumes and doses of empty and full bladder states. The Mann-Whitney $U$ test was also engaged to compare volumes or dose values in independent patient groups. All $p$ values were two-sided, with statistical significance set at $p<0.05$.

\section{Results}

Median age of the 15 patients analyzed was 58 years (range, 38-81 years). Eight patients (53\%) had stage IA endometrial cancer, and seven patients $(47 \%)$ had stage IB disease. As demonstrated in Table 1, all target and OAR volumes (except bladder) were similar. Typically, small bowel was displaced anteriorly and superiorly from the vaginal cylinder applicator (shown in Fig. 1), significantly
Table 1. Target volume and organ-at-risk volumes in full and empty bladder plans

\begin{tabular}{lccc} 
Volume & $\begin{array}{c}\text { Empty bladder } \\
(\mathrm{cc} \pm \mathrm{SD})\end{array}$ & $\begin{array}{c}\text { Full bladder } \\
(\mathrm{cc} \pm \mathrm{SD})\end{array}$ & $p$ \\
\hline CTV & $99.4 \pm 21.5$ & $100.4 \pm 22.3$ & 0.27 \\
\hline Rectum & $81.3 \pm 31.7$ & $83.9 \pm 39.1$ & 0.31 \\
\hline Bladder & $55.0 \pm 14.4$ & $255.1 \pm 32.3$ & $<0.001$ \\
\hline Sigmoid & $59.9 \pm 29.7$ & $63.5 \pm 30.2$ & 0.76 \\
\hline Intestine & $348.5 \pm 107.6$ & $337.9 \pm 104.6$ & 0.27
\end{tabular}

CTV-clinical target volume
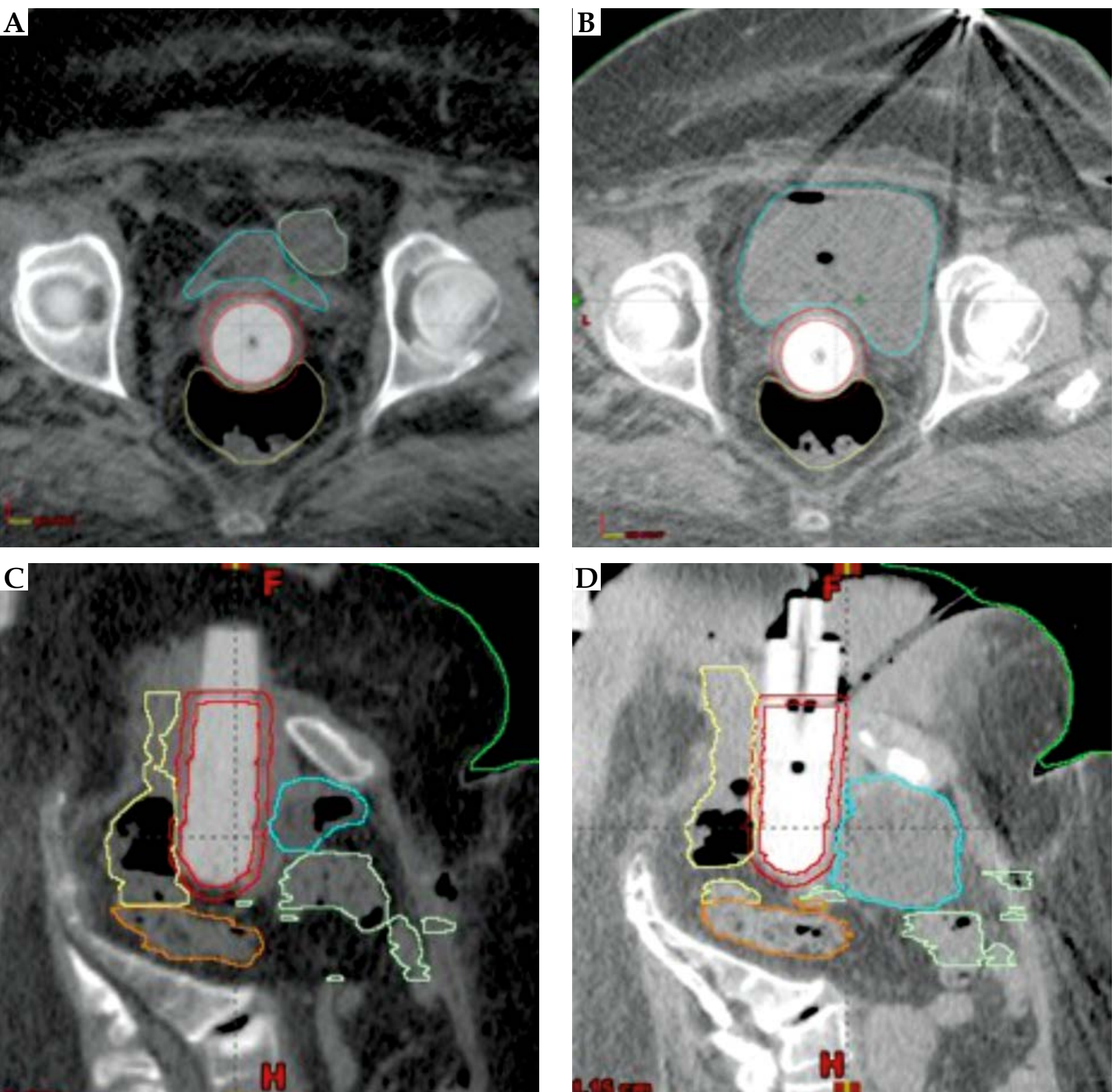

Fig. 1. Organs at risk (OARs) in axial plane with bladder empty (A) and full (B) (small bowel displaced by vaginal cylinder applicator); Vaginal cylinder (relative to OARs) in sagittal plane with bladder empty (C) and full (D) (small bowel displaced anteriorly and superiorly but stable posteriorly) 

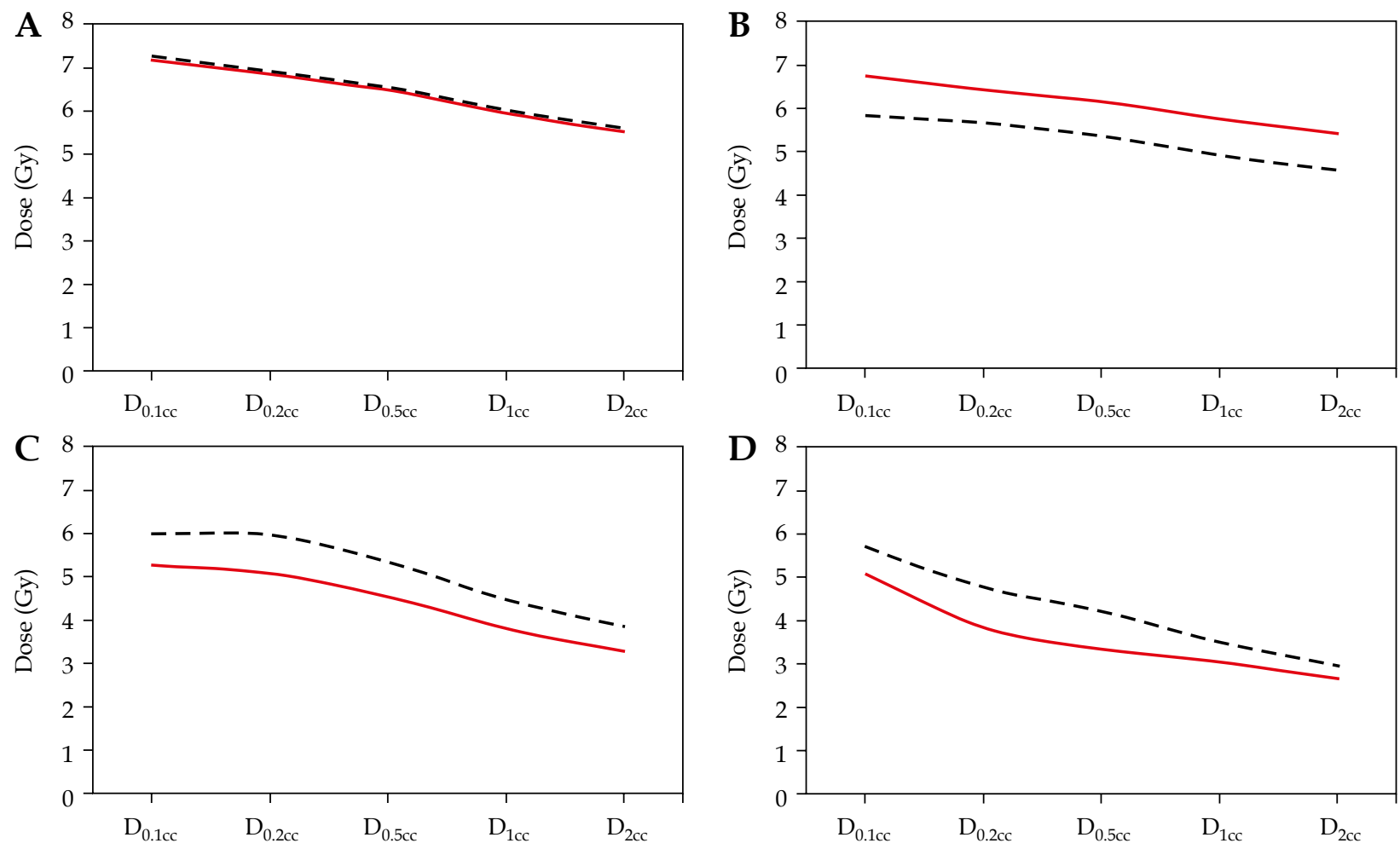

Fig. 2. Dose-volume histograms with bladder empty (dashed line) and full (red line): (A) rectum, (B) bladder, (C) sigmoid colon, and (D) small bowel

increasing mean cylinder-to bowel distance $(1.69 \mathrm{~cm}$ vs. $1.20 \mathrm{~cm} ; p=0.006$ ).

Bladder distention had no impact on dosimetry of rectum (Fig. 2A). However, the effects on bladder (Fig. 2B), sigmoid colon (Fig. 2C), and small bowel (Fig. 2D) dosimetry were appreciable. With a full bladder, mean $\mathrm{D}_{2 \mathrm{cc}}$ of bladder significantly increased from 4.55 Gy to $5.40 \mathrm{~Gy}$ (18.7\% gain; $p<0.001)$, and reductions in mean $\mathrm{D}_{2 \mathrm{cc}}$ values of sigmoid colon (15.1\%) and small bowel (10.5\%) neared statistical significance (Table 2).

$\mathrm{D}_{50 \%}$ values of rectum and bladder were unaffected by bladder distention (Table 3), and declines seen in mean $\mathrm{D}_{50 \%}$ values of sigmoid colon $(22.7 \%, p=0.12)$ and small bowel $(19.0 \%, p=0.13)$ again fell short of statistical significance.

\section{Discussion}

In this study, we determined that the combination of a distended bladder and an empty rectum prior to BRT significantly increases bladder doses, with decreases in sigmoid colon and small bowel exposures that neared statistical significance and no impact on rectal dosimetry.

Based on PORTEC-II study outcomes, adjuvant vaginal vault BRT has been accepted as standard therapy in early-stage endometrial cancer. Unfortunately, earlier methods of BRT planning involved orthogonal films, restricting doses to a $5 \mathrm{~mm}$ distance from cylinder. In addition, doses to rectum and bladder were perhaps inaccurately gauged by ICRU reference points. Although the chief toxicities for vaginal cuff BRT are appropriate to be intestinal, due to close proximity of the vaginal cylinder, conventional plans provide no reference points for defining doses to small bowel. Hence, determining OAR exposures through CT-based planning is of particular interest.

CT-based plans have been routinely used in cervical cancer BRT, where target volumes are managed with comparatively greater sophistication. A US survey indicates that most centers do not routinely document OAR doses during adjuvant vaginal cuff BRT after hysterectomy [3]. Holloway et al. [18] investigated the need for CT-based treatment planning on each insertion of vaginal vault BRT, and found that doses to adjacent organs did not vary significantly between fractions. Nevertheless, an awareness of collateral exposures is needed to ensure that side effects of such therapy, given as prophylactic treatment, are not excessive.

In order to reduce OAR doses, the impact of bladder distention has been investigated. Results of this strategy in patients with cervical cancer have conflicted, most reporting marginal differences in dosing decrements [12-14]. Few researchers have examined the impact of bladder distention on dose to OARs in patients treated post-hysterectomy with HDR vaginal cuff BRT $[15,16,19,20]$. Hoskin and Vidler [19] found that $100 \mathrm{ml}$ bladder infusions reduced exposure of small bowel within the high-dose treatment region (as measured on CT slices through cranial-most dwell positions) by $57.5 \%$, compared with a voided bladder. At the same time, mean maximal bladder dose did not change significantly. Of note, values were based on 2D measurements of bladder height; thus, volumetric parameters of bladder were not assessed, and doses to sigmoid colon and small bowel were not evaluated. Kobzda et al. [15] found the dose to 
Table 2. Mean $\mathrm{D}_{2 c \mathrm{c}}$ of organs at risk in empty and full bladder plans

\begin{tabular}{lcccc} 
Organs & Empty bladder (Gy \pm SD) & Full bladder (Gy \pm SD) & \% Change & $p$ \\
\hline Rectum & $5.51 \pm 1.01$ & $5.59 \pm 1.01$ & +0.5 & 0.61 \\
\hline Bladder & $4.55 \pm 0.72$ & $5.40 \pm 1.22$ & +18.7 & $<0.001$ \\
\hline Sigmoid & $3.83 \pm 2.00$ & $3.25 \pm 1.27$ & -15.1 & 0.11 \\
\hline Bowel & $2.94 \pm 1.72$ & $2.63 \pm 1.88$ & -10.5 & 0.14
\end{tabular}

Table 3. Mean $D_{50 \%}$ of organs at risk in empty and full bladder plans

\begin{tabular}{|c|c|c|c|c|}
\hline Organs & Empty bladder $(\mathrm{Gy} \pm \mathrm{SD})$ & Full bladder $(\mathrm{Gy} \pm \mathrm{SD})$ & $\%$ Change & $p$ \\
\hline Rectum & $3.51 \pm 1.61$ & $3.34 \pm 1.85$ & -4.8 & 0.33 \\
\hline Bladder & $2.92 \pm 1.23$ & $3.05 \pm 2.28$ & +4.5 & 0.69 \\
\hline Sigmoid & $1.98 \pm 1.53$ & $1.53 \pm 0.98$ & -22.7 & 0.12 \\
\hline Bowel & $1.53 \pm 0.64$ & $1.24 \pm 0.50$ & -19.0 & 0.13 \\
\hline
\end{tabular}

the empty bladder is lower than when the bladder is full (4.6 Gy (range: 3.1-5.6 Gy) vs. 4.9 Gy (range: 3.9-5.9 Gy); $p<0.05)$, and the doses to the bowels increase proportionally in the empty state of the bladder comparing to the full organ (4.6 Gy (range: 2.5-7.3 Gy) vs. $4.1 \mathrm{~Gy}$ (range: 1.3-5.7 Gy); $p<0.05$ ).

When Stewart et al. [20] examined the effects of bladder distention on BRT doses to bladder, rectum, and urethra using 3D image-based treatment planning, they found a correlation between midline maximal bladder point dose and maximal rectal point dose. Their conclusion was that maximal bladder point and maximal rectal point were acceptable surrogates of $D_{2 c c}$ volumetric assessment. Similar to our findings, Stewart et al. [20] reported that bladder distention increased the $\mathrm{D}_{2 \mathrm{cc}}$ of bladder, with no appreciable change in $\mathrm{D}_{2 \mathrm{cc}}$ of rectum. Dosimetry of sigmoid colon and small bowel, were not evaluated, but they too documented a significant increase in cylinder-to-bowel distance $(0.57 \mathrm{~cm}$ to $1.16 \mathrm{~cm} ; p=0.002)$ with filling of the bladder. In our study, cylinder-to-bowel distance increased from $1.20 \mathrm{~cm}$ to $1.69 \mathrm{~cm}(p=0.006)$.

Hung et al. [16] also evaluated OAR doses to rectum, bladder, sigmoid colon, and small bowel. As reported, a full bladder resulted in a significant reduction in mean $\mathrm{D}_{2 \mathrm{cc}}$ of small bowel (677 to $408 \mathrm{cGy} ;-39.7 \%$ ) and $\mathrm{D}_{50 \%}$ of small bowel (168 to $132 \mathrm{cGy} ;-21.4 \%$ ). Corresponding $\mathrm{D}_{2 \mathrm{cc}}$ and $\mathrm{D}_{50 \%}$ doses to rectum and to sigmoid colon were unaffected by bladder distention. Our detailed dosimetric assessment was at odds with these findings, showing instead that bladder distention significantly increases doses to bladder, whereas sigmoid colon and small bowel exposures declined to near significant levels.

Above differences may be attributed to the inherent uncertainties in measuring exceedingly small distances of select regions in mid-sagittal imaging plane. Variance in full bladder volumes between studies also may have contributed to disparities. In the study conducted by Stewart et al. [20], a full bladder was defined as the volume achieved by a $32 \mathrm{oz}$ intake of water 1 hour prior to cylinder insertion. Similarly, Kobzda et al. [15] provided a sufficient bladder filling by asking to consume $400 \mathrm{ml}$ of water 40 minutes before the CT scans were taken. On the other hand, Hung et al. [16] defined a full bladder as a $180 \mathrm{ml}$ infusion of sterile water.

We performed two CT scans, one with the bladder emptied by Foley catheter, and one following retrograde infusion of saline $(180 \mathrm{ml})$ via Foley catheter. Mean full bladder volumes of $32 \mathrm{US} \mathrm{fl} \mathrm{oz}(946 \mathrm{ml})$ for Stewart et al. [20], $235.0 \mathrm{ml}$ for Hung et al. [16], $229.7 \mathrm{ml}$ for Kobzda et al. [15], and $255.1 \mathrm{ml}$ in the current study were subsequently recorded. Another reason of having larger bladder volume in Stewart et al. and our study is, in both studies urinary catheter was used to inflate bladder, compared to other studies. A larger bladder expanse may displace small bowel to a greater extent, thus explaining the increases in cylinder-to-bowel distance registered by Stewart et al. [20] and in our work.

Our dosimetric study has some limitations, primarily, the limited number of patients recruited, both of which curtail any generalization of results. Furthermore, without standardization of bladder filling it is difficult to issue recommendations for filling of the bladder during vaginal vault BRT. However, our study does underscore that bladder distention may cause unwanted increases in bladder doses, while decreasing doses to sigmoid colon and small bowel. Finally, our investigation was a dosimetric study by design, devoid of clinical outcomes. Further clinical investigation with extended follow-up is needed to determine its practical merit.

\section{Conclusions}

This dosimetric study illustrates that the combination of a distended bladder and an empty rectum prior to BRT of vaginal vault may cause significant increases in bladder doses, while possibly lowering doses to sigmoid colon and small bowel. Because the impact of bladder distention on gastrointestinal and genitourinary toxicities has yet to be demonstrated, a suitable bowel prep before each therapeutic session may suffice (especially in 
high-volume patient clinics), with no need to inflate the bladder. The core concept here is tentative, requiring further dosimetric and clinical corroboration.

\section{Disclosure}

Authors report no conflict of interest.

\section{References}

1. Amant F, Mirza MR, Creutzberg CL. Cancer of the corpus uteri. Int J Gynaecol Obstet 2012; 119 Suppl 2: S110-117.

2. Aalders J, Abeler V, Kolstad P, Onsrud M. Postoperative external irradiation and prognostic parameters in stage I endometrial carcinoma: clinical and histopathologic study of 540 patients. Obstet Gynecol 1980; 56: 419-427.

3. Small W Jr, Beriwal S, Demanes DJ et al. American Brachytherapy Society consensus guidelines for adjuvant vaginal cuff brachytherapy after hysterectomy. Brachytherapy 2012; 11: 58-67.

4. Nout RA, Smit VT, Putter H et al. Vaginal brachytherapy versus pelvic external beam radiotherapy for patients with endometrial cancer of high-intermediate risk (PORTEC-2): an open-label, non-inferiority, randomised trial. Lancet 2010; 375: 816-823.

5. Nout RA, Putter H, Jürgenliemk-Schulz IM et al. Quality of life after pelvic radiotherapy or vaginal brachytherapy for endometrial cancer: first results of the randomized PORTEC-2 trial. J Clin Oncol 2009; 27: 3547-3556.

6. Kim H, Kim H, Houser C, Beriwal S. Is there any advantage to three-dimensional planning for vaginal cuff brachytherapy? Brachytherapy 2012; 11: 398-401.

7. Onal C, Arslan G, Topkan E et al. Comparison of conventional and CT-based planning for intracavitary brachytherapy for cervical cancer: target volume coverage and organs at risk doses. J Exper Clin Cancer Res 2009; 28: 95.

8. Kim RY, Shen S, Duan J. Image-based three-dimensional treatment planning of intracavitary brachytherapy for cancer of the cervix: dose-volume histograms of the bladder, rectum, sigmoid colon, and small bowel. Brachytherapy 2007; 6 : 187-194.

9. Caon J, Holloway C, Dubash R et al. Evaluating adjacent organ radiation doses from postoperative intracavitary vaginal vault brachytherapy for endometrial cancer. Brachytherapy 2014; 13: 94-99.

10. Haie-Meder C, Pötter R, Van Limbergen E et al. Recommendations from Gynaecological (GYN) GEC-ESTRO Working Group (I): concepts and terms in 3D image based 3D treatment planning in cervix cancer brachytherapy with emphasis on MRI assessment of GTV and CTV. Radiother Oncol 2005; 74: 235-245.

11. Pötter R, Haie-Meder C, Van Limbergen E et al. Recommendations from gynaecological (GYN) GEC ESTRO working group (II): concepts and terms in 3D image-based treatment planning in cervix cancer brachytherapy-3D dose volume parameters and aspects of 3D image-based anatomy, radiation physics, radiobiology. Radiother Oncol 2006; 78: 67-77.

12. Cengiz M, Gürdalli S, Selek U et al. Effect of bladder distension on dose distribution of intracavitary brachytherapy for cervical cancer: three-dimensional computed tomography plan evaluation. Int J Radiat Oncol Biol Phys 2008; 70: 464-468.

13. Sun LM, Huang HY, Huang EY et al. A prospective study to assess the bladder distension effects on dosimetry in intracavitary brachytherapy of cervical cancer via computed tomography-assisted techniques. Radiother Oncol 2005; 77: 77-82.
14. Kim RY, Shen S, Lin HY et al. Effects of bladder distension on organs at risk in 3D image-based planning of intracavitary brachytherapy for cervical cancer. Int J Radiat Oncol Biol Phys 2010; 76: 485-489.

15. Kobzda JD, Cikowska-Wozniak E, Michalska M, Makarewicz R. Three-dimensional dosimetry of the full and empty bladder in high dose rate vaginal cuff brachytherapy. Int J Gynecol Cancer 2014; 24: 923-927.

16. Hung J, Shen S, De Los Santos JF, Kim RY. Image-based 3D treatment planning for vaginal cylinder brachytherapy: dosimetric effects of bladder filling on organs at risk. Int J Radiat Oncol Biol Phys 2012; 83: 980-985.

17. Onal C, Topkan E, Efe E et al. Comparison of rectal volume definition techniques and their influence on rectal toxicity in patients with prostate cancer treated with 3D conformal radiotherapy: a dose-volume analysis. Radiat Oncol 2009; 4: 14.

18. Holloway CL, Macklin EA, Cormack RA, Viswanathan AN. Should the organs at risk be contoured in vaginal cuff brachytherapy? Brachytherapy 2011; 10: 313-317.

19. Hoskin PJ, Vidler K. Vaginal vault brachytherapy: the effect of varying bladder volumes on normal tissue dosimetry. $\mathrm{Br}$ J Radiol 2000; 73: 864-866.

20. Stewart AJ, Cormack RA, Lee $\mathrm{H}$ et al. Prospective clinical trial of bladder filling and three-dimensional dosimetry in high-dose-rate vaginal cuff brachytherapy. Int J Radiat Oncol Biol Phys 2008; 72: 843-848. 